\title{
Subject Index to Volume 22
}

Achondrogenesis, type II, extracellular matrix abnormalities. 324

Acid base balance, effect of maternal hyperglycemia on, fetal lamb. 355

Acidosis, metabolic, during streptococcal group B sepsis. piglet. 509

Adrenocorticotropin, response to corticotropin-releasing factor, during pubertal development. 41

Airway, occlusion, genioglossus response. apneic versus nonapneic infants. 68.3

I-Amino acids, branched chain. catabolism compared, cultured skin fibroblasts. 591

Anaphylaxis, pertussis toxin-dependent. mouse, 262

Antitrysin, alpha-1, concentration in human milk, 268

Aorta, thoracic, coarctation, renal perfusion

Apnea after, puppy, 445

airway occlusion, genioglossus response, apncic versus nonapneic infants, 68.3

short, types and frequency, developmental study, term and preterm infants. 344

Arginine, effect on creatine excretion, hyperornithinemia-hyperammonemiahomocitrullinuria syndrome, 364

Asphyxia, perinatal, cerebral survival after. newborn sheep, 62

Atrioventricular node, function, immature heart, dog, 616

Behavior, fetus, 30 to 32 weeks gestation. 655 Betamethasone

effect on neutrophil chemotaxis, neonate. 150

pulmonary compliance increased by, preterm rabbit. 730

Bilirubin, effect on cerchral metabolism. newborn piglet. 135

Bipyridine, derivatives, arteriolar resistance

Birth lowered by, neonatal lamb, 422

related events

blood flow distribution and, 6.34

effect on central blood flow patterns, 557

Blood flow

central, birth-related events and patterns of 557

distribution, birth-related events and, 6.34

intracranial arterial, early postnatal changes, term infants, 46

regional, cyanotic heart discase, neonatal lamb, 389

Body water, estimated from total body electrical conductivity measurements, infants, 417

Bone status, assessment, transmission ultrasonics used for neonate. 541

Bovine serum albumin. immunization, anaphylactic reaction. mousc, 262

Brain

cerebral arteries, developmental changes and hypoxic sensitivity, newborn lamb, 192

cerebral metabolism, effect of bilirubin and sulfisoxazole, newborn piglet. 135 oxidative metabolism, head position and preterm and term neonates. 302

postasphyxial cerebral survival, after oxygen free radical scavengers and calcium antagonist treatment. newborn sheep. 62

Breath test, cholestervl octanoate. pancreatic insufficiency detected by, rat, 257

Bronchiolitis. respiratory mechanics. salbutamol effect, infants. 83

Brush border membranes, dipeptide transport. glveylsareosine and. rat kidney. 641

Calcium

dietary variations. effect on renal and intestinal calcium-binding proteins, 518

homeostasis, role of magnesium, neonate. 319

response to serum melatonin, neonatal rat, 414

transport, developmental maturation. rat. 173

Calcium antagonist, asphyxia treatment. cerebral survival after. newborn sheep. 62

Cardiac output. intravenous catecholamine effect. unanesthetized hypoxemic newborn lambs, 667

Carnitine, metabolism, in valproate-treated rats, efiect of I-carnitine supplementation. 497

(r-Cascin, local antibodies to. in infant saliva. 399

Catecholamines

endogenous, comparison, renal $\beta$-adrenoceptor vasodilation. sheep. 465

intravenous. cardiac output and, unanesthetized hypoxemic newborn lambs. 667

Cercbrospinal fluid, cortical subarachnoid. ventilation pressure effect on prostanoids in, newborn pig. 647

Chediak-Higashi syndrome. neutrophils. normal-sized lysosomes in. 208

Chlorpromazine, peroxisomal proliferation caused by, heart and liver, mouse, 748

(Cholesterol, deprivation, alterations in small intestine after. piglet, 330

Cholesteryl octanoate, breath test, pancreatic insufficiency detected by, rat. 257

Chromatid. sister exchange. vitamin $\mathrm{K}_{1}$ and. human leukocytes and fetal sheep cells. $4(05$

(itrulline. effect on creatine excretion. hy perornithinemia-hyperammonemiahomocitrullinuria sındrome. 364

Collagen

lung maturation related to, fetal sheep. 339 ontogenesis. in fetal lung. sheep. 335

Colon, neonatal, short chain fatty acid protile by fecal water analysis, pig. 72(

Complement

activation. effect of Hacmophilus influchzae type b lipopolysaccharide on, 650

( 3 converting factor. meningococcal septicemia and. case report. 123

Corticotropin-releasing factor, adrenocorti- cotropin, eortisol, and dehvedroepiandrosterone response, during pubertal development. 41

cortisol, response to corticotropin-releasing factor. during pubertal development. 41

Cranium, irradiation. role of growth hormone in stunted head growth after 402

Cyclooxygenase, effect on pulmonary function. group B streptococcal sepis, piglet. 478

(ysteine. levels developing kidney, rat, 605

(ystic tibrosis

altered antibody isotype in. respense to polvsaccharide antigens. $7(28$

heterozygotes. sweat hyposecretion in. 271

mucoid Psedudomonas acruginesed, suscep) tibility to nonopsonic phagocritosis 429

Psendemonas acruginosa pulmonary in fection, immunohistological cvaluation. 74.3

serum opsonic activity, corrclation with colonization and discase state, 383

tracheobronchial mucin, biochemical propertics. 54.

Dehydroepiandrosterone. response to corticotropin-releasing factor, during pubertal development, 41

Dexamethasone, somatomedin C/insulinlike growth factor I and, in insulin resistance, 188

Diaphragm, muscle fiber type, development. swine. 449

Diarrhea, rotavirus, protein transport during mice. 72

Dict

calcium variations. effect on renal and in testinal calcium-binding protcins. 518

high fat peroxisomal proliferation caused by, mouse heart and liver, 748

Dipeptide, transport, renal brush border membranes, rat, 641

D.NA, protein synthesis and, developing rat pancreas, 34

Docasahexaenoic acid. uptake, related to in take and mode of administration, preterm infants. 29?

Down svindrome, humoral and eell-mediated immunity, age-related changes, 536

Drugs, nonsteroidal antiinflammatory, constriction of ductus arteriosus by, tetal rat. 567

Ductus arteriosus, constriction, nonsteroidal antiinflammatory drugs and, tetal rat. 567

Ficosapentaenoic acid, uptake, related to in take and mode of administration, preicrm infants. 292

Ehastin

lung maturation related to, fetal sheep, 3.39 ontogenesis, in fetal lung, sheep, 3.35

Electrical conductivity, total bods, fat-fice mass and total body water estimated from, infants. 417 
Embryo, dorsal aortic flow, effect of heart rate increase, chick, 442

Enterectomy, proximal, cellular adaptation of small intestine after, rat, 29

Enzyme

cell type-specific reactivities, ontogeny, in kidney collecting ducts, 504

microvillous, after proximal enterectomy, 29

protein, absence, in tyrosinemia, 394

Erythrocyte

membrane fluidity, changes, obese children, 141

prolidase subunit absent in, patient with prolidase deficiency, 627

Escherichia coli, adherence to intestinal tract, inhibition by human milk, guinea pig, 130

Estradiol-17 $\beta$, effect on lung maturation, ovine fetus, 145

Estrogen, effect on lung maturation, ovine fetus, 145

Ethyl 2[5(4-chhorophenyl)pentyl]oxiran-2carboxylic acid, peroxisomal proliferation caused by, mouse heart and liver, 748

European Society for Paediatric Gastroenterology and Nutrition, abstracts of annual meeting, 96

European Society for Pediatric Research, abstracts of annual meeting, 217

Eye, vitamin E disposition in, cats, 16

Fat, absorption, taurine supplementation effect, preterm infants, 67

Fat-free mass

estimated from total body electrical conductivity measurements

infants, 417

Fatty acid, short chain, fecal water analysis, colon, newborn pig, 720

Fetus

acid base balance and lung liquid production, effect of maternal hyperglycemia on, lamb, 355

atrial natriuretic polypeptide in, ontogeny and characterization, rat, 115

behavior, 30 to 32 weeks gestation, 655

cardiac output, insulin effect, lamb, 168

circulation, leukotrienes and, 350

cyclic adenosine monophosphate content, milrinone effect, rabbit, 282

development, transforming growth factor role, sheep, 609

ductus arteriosus constriction, nonsteroidal antiinflammatory drugs and, rat, 567

early development, structure and abundance of cartilage proteoglycans during, 409

growth and development, maternal hyperinsulinemia and, rat, 432

heart, development of myocardial contractile system, rabbit, 201

heart and great vessels, in situ morphology, rat, 573

hypotrophic, serum growth-promoting activity, 39

insulin-like growth factor I concentration, glucocorticoid effect, rat, 92

intravenous nutritional supplementation, effect on growth retardation, sheep, 55

kidney, action of atrial natriuretic peptide, sheep, 11

lactate metabolism, perfused heart, pig, 552 lung

elastin and collagen ontogenesis, sheep, 335

maturation, elastin and collagen related to, sheep, 339

maturation, estradiol- $\beta$ effect, sheep, 145

temporal linkage of glycogen and saturated phosphatidylcholine, type II cells, rat, 79

myocardial mechanical function, milrinone effect, rabbit, 282

plasma, $\alpha 1$-protease inhibitors in, mice, 1

protein synthesis and leucine oxidation, ontogenetic changes in rates of, 688

small intestine, damage prevented by mannan and other sugars, rat, 703

somatomedin immunocytochemistry in, 245

utilization of metabolites by, fed and starved rats, 6

ventricle, response to filling and arterial pressure and to in utero ventilation, lamb, 621

vitamin $\mathrm{K}$ deficiency, mutagenic events reduced by, 405

Fibroblasts, cultured human skin, catabolism of branched-chain L-amino acids in, 591

Genioglossus, response to airway occlusion, apneic versus nonapneic infants, 683

Germinal matrix, quantitative analysis, intracerebral hemorrhage and, newborn puppy, 23

Glucocorticoids, effect on plasma insulin-like growth factor I concentration, rat fetus, 92

Glucose, metabolism, placenta, effect of insulin, glucose, and ketone bodies, rat, 483

Glutathione, levels, developing liver, rat, 605

Glycogen, saturated phosphatidylcholine and, fetal lung type II cells, rat, 79

Glycosylation, intestinal, developmental changes, rat, 250

Glycylsarcosine, dipeptide transport and, renal brush border membranes, rat, 641

Great vessels, in situ morphology, fetal and newborn rat, 573

Growth and development

atrioventricular nodal function, immature heart, dog, 616

developing kidney and liver, cysteine and glutathione levels, rat, 605

diaphragmatic muscle fiber type, swine, 449

humoral and cell-mediated immunity, agerelated changes, Down syndrome, 536 intestinal glycosylation and, rat, 250

maternal hyperinsulinemia and, fetal rat, 432

postrest potentiation in atria, effects of postnatal maturation, 524

short apneas, types and frequency, term and preterm infants, 344

somatomedin $\mathrm{C} /$ insulin-like growth factor I and, in insulin resistance, 188

transforming growth factor role, fetal development, sheep, 609

Growth cartilage, achondrogenesis type II, extracellular matrix abnormalities, 324

Growth hormone

molecular variants of, children, 21

role in stunted head growth, after cranial irradiation, 402
Growth retardation, embolization-induced, fetal nutritional supplementation and, sheep, 55

Growth sparing, biochemical determinants, during nutritional deprivation or enhancement, neonate, 599

Haemophilus influenzae

antibiotic-resistant, plasmid-mediated, infant, 438

type $b$, lipopolysaccharide effect on complement activation and polymorpho-

Head nuclear leukocyte function, 659

growth, after cranial irradiation, role of growth hormone, 402

position, brain oxidative phosphorylation

Heart and, preterm and term neonates, 302

cardiac output, 'nsulin effect, fetal lamb, 168

disease, cyanotic, regional blood flow and oxygen delivery, neonatal lamb, 389

immature, atrioventricular nodal function, dog, 616

in situ morphology, fetal and newborn rat, 5.73

lactate metabolism, isolated, perfused fetal, and neonatal, pig, 552

left ventricular function, effect of bipyridine derivatives, neonatal lamb, 422

myocardial contractile system, development, fetal rabbit, 201

peroxisomal proliferation

chlorpromazine and, mouse, 748

high fat diet and, mouse, 748

propafenone hydrochloride effects, neonatal canine, 50

rate

increase, effect on dorsal aortic flow, chick embryo, 442

variability during respiratory pauses, puppies and dogs, 306

Heart atria, postrest potentiation, effects of postnatal maturation on, rabbit, 524

Heart ventricle, response to filling and arterial pressure and to in utero ventilation, fetal lamb, 621

Helium, pulmonary clearance, effect of acute hemorrhagic hypotension, rabbit, 595

Hemorrhage, germinal matrix vessels and, newborn puppy, 23

Howland Award

acceptance, 761

presentation, 760

Human milk

antibodies, infant saliva, 399

alpha-1-antitrypsin concentration, 268

Escherichia coli adherence inhibited by, intestinal tract, guinea pig, 130

future research, 493

tuberculin immunity transferred via, 277

vitamin $\mathrm{K}_{1}$ content, stage of lactation and, 513

Hyperglycemia, maternal, effect on acid base balance and lung liquid production, fetal lamb, 355

Hyperinsulinemia

in leprechaunism, infant, 286

maternal, fetal growth limited by, rat, 432

Hyperornithinemia-hyperammonemia-homocitrullinuria syndrome, creatine excretion, effect of citrulline, arginine, or ornithine supplement, 364

Hypotension, acute hemorrhagic, effect on pulmonary clearance of helium in- 
stilled in colon, rabbit. 595

Hypoxemia

arousal from slecp during, lamb. 471

oxygen transport and metabolism during. lamb, 177

Hypoxia

alveolar, small pulmonary wedge pressure increased by, awake lamb, 679)

cerebral arteries and. newborn lamb. 192

Immunity, humoral and cell-mediated, agerelated changes, Down syndrome. 536

Immunization

bovine serum albumin, murine responses, 262

pertussis toxin, murine responses, 262

Immunoglobulin

effect on sepsis and meningitis, neonatal rat. 455

secretory component, after proximal enterectomy, 29

Indomethacin, in utere, effects on pulmonary vasculature, newborn guinea pig. 693

Infant

see also Neonatc

airway occlusion. genioglossus response. apncic versus nonapneic infants, 683

bacterial meningitis, plasmid-mediated resistance, 438

bronchiolitis, salbutamol effect, 8.3

fat-free mass and total body water. estimated from total body electrical conductivity measurements, 417

intracranial arterial blood flow velocities, early postnatal changes, 461

leprechaunism, in vitro insulin resistance, 286

pyruvate dehydrogenase, systemic deficiency, 312

saliva, local antibodies to $\alpha$-casein and $\beta$ lactoglobulin in. 399

tuberculin immunity, transfer via breast milk, 277

Infant formula, taurine supplementation, fat absorption improved by, preterm infants, 67

Infection, pulmonary. Pseudomonas aeruginosa and Pserudomonas cepacia. mouse, 698

Insulin, cardiac output and, fetal lamb, 168

Insulin-like growth factor, immunocytochemistry, human fetus, 245

Insulin-like growth factor I, concentration. glucocorticoid effect, rat fetus, 92

Insulin resistance. impaired synergism between somatomedin (/insulin-like growth factor I and dexamethasone in patient with, 188

Intestinal tract

bacterial adherence, inhibition by human milk, guinea pig. 130

brush border membrane vesicles, developmental maturation of calcium transport by, 173

surface acid microclimate, developmental aspects, 497

Iron, absorption, lactoferrin and, neonate. 651

Kidney

action of atrial natriuretic peptide, ovine fetus, 11

brush border membranes, dipeptide transport in. rat, 641

collecting ducts, ontogeny of cell type-specific reactivities in. 504 decreased perfusion, after correction of coarctation, puppy. 445

developing. crsteine and glutathione levels. rat. 605

ontogeny of $\beta$-adrenoceptor-mediated vasodilation, endogenous catecholamines compared, sheep, 465

taurine transport. fluidity and phospholipids. voung rat. 163

Lactate

metabolism. isolated. perfused fetal. and neonatal pig heart. 552

production. fed and starved rat fetuses. 6

Lactoferrin. neonatal iron absorption and. 651

$\beta$-I actoglobulin, local antibodies to. in infant saliva. 399

Larynx. muscle activity control. preterm infants. 87

Latin American Society for Pediatric Research

abstracts of 24 th annual meeting. 368

author index of abstracts. 374

lecithin/sphingomvelin ratio. determination. tracheal aspirates. neonates. 154

I.eprechaunism. in vitro insulin action. infant. 286

Leucine. oxidation, ontogenetic changes in rates of. fetus. 688

Leukocyte

polymorphonuclear

abnormalities, neonate. 375

effect of Hacmophilus influenzue type b lipopolysaccharide on. 659

Leukotriene, putative synthesis inhibitor. fetal circulation and. lamb, 350

l.ipid, infusion, lung vascular effects. awake lamb. 714

I ipopolysaccharide. Hacmophilus in/luenzas type b. effect on complement activation and polymorphonuclear leukocyte function. 659

I.ipoprotein lipase. inhibition, in steroid responsive nephrotic syndrome. 197

Lipoxygenase, effect on pulmonary function. group B streptococcal sepsis. piglet. 478

Liver

developing. cysteine and glutathione levels. rat. 605

peroxisomal proliferation

chlorpromazine and. mouse. 748

high fat diet and. mouse. 748

Lung

blood flow. leukotrienes and. fetal lamb. 350

chronic Pseudomonas infections. mouse. 698

development. neonatal protein deprivation and. rat. 45

clastin and collagen ontogenesis. fetal sheep. 3.35

fetal maturation. estradiol-17/8 effect. sheep. 145

function

group B streptococcal sepsis, piglet. 478 tachypnea of unknown cause. neonate. 581

lipid infusion, vascular effects, awake lamb. 714

liquid production, effect of maternal hyperglyeenia. fetal lamb. 355

maturation. elastin and collagen related to. sheep fetus. 339

neonatal

effect of indomethacin in utero, guine: pig. 693

impaired pulmonary clearance of pneumococci. rat. 736

premature. betamethasone effect on pulmonary compliance, rabbit. 7.30

Psoudomonas acruginesia infection. immunohistological evaluation. cystic librosis. 74.3

type II cells. temporal linkage of glveogen and phosphatidyleholine, rat. 79

Macromolecules

biochemical determinants of growth sparing and. during nutritional deprivaltion or enhancement, neonatal brain and heart. 590)

intestinal absorption, during viral enteritis. mice. 72

Magnesium, role in calcium homeostasis. neonate. 319

Mannan, small intestine damage prevented bx. rat fetus. 703

Mannitol. experimental meningitis treatment. Ha'mophilus influenzac type b. rabbit. 118

Maturation, postnatal. effects on postrest potentiation, rabbit atria. 524

Melatonin. photic and pharmacologic manipulation. calcemic responses, neonatal rat. 414

Membrane. fluidity, nonmuscke cells, Duchenne muscular dystrophy: 488

Meningiti

bacterial, plasmid-mediated resistance, infant 438

Excherichia coli, immunoglobulin effect. neonatal rat. 455

Hacmophilus influenzae tvpe b, mannitol treatment. rabbit. 118

Metabolism, uterine, late pregnancy, pig, 587

Metabolite, utilization, fetuses from fed and starved rats, 6

Midpregnancy. serum growth-promoting activity, normal and hypotrophic fituses. 39

Milk feed. composition. upper intestinal mucosa proliferation and. newborn guinea pig. 67.5

Milrinone. effect on myocardial mechanicat function and erclic adenosine monophosphate content. Retal rabbit. 282

Mitochondria. inhibition of function bs margosa oil. Reve's syndrome and. rat. 184

Molecule, new functions, presidential akdress. 755

Mother, tuberculin immunity, transfer to infant. 277

Mucin, tracheobronchial, biochemical properties. cystic fibrosis. 545

Mucosa, upper intestinal. milk feed composition and, newborn guinea pig. 675

Muscle. laryngeal. activity control. preterm infints. 87

Muscular dystrophy. Duchenne, membrane fluidity of nonmuscie cells. 488

Veonate

arteriolar resistance, effece of hipuridine derivatives on lamb. 42

bone status. assessment by transmission ultrasonics. 541

brain. developmental changes and hepoxic sensitivity, lamb cerebral arteries, 192

calcium homeostasis, role of magnesium, 310 
cardiac output, intravenous catecholamine effect, unanesthetized hypoxemic lambs

cerebral metabolism, effect of bilirubin and sulfixazole, piglet, 135

cerebral vessels, quantitative analysis, dogs, 23

cholesterol deprivation, small intestine alterations after, piglet, 330

cyanotic heart disease, regional blood flow and oxygen delivery, lamb, 389

growth sparing, biochemical determinants, during nutritional deprivation and enhancement, 599

heart, propafenone hydrochloride effects, dog, 50

heart and great vessels, in situ morphology, rat, 573

hypoxemia, arousal from sleep during, lamb, 471

impaired pulmonary clearance of pneumococci, rat, 736

iron absorption in, lactoferrin and, 651

lactate metabolism, pig heart, 552

lecithin/sphingomyelin ratio determination, tracheal aspirates, 154

neutrophil chemotaxis, betamethasone effect, 150

polymorphonuclear leukocytes, biochemical, structural, and functional abnormalities, 375

protein deprivation, lung development impaired by, rat, 45

pulmonary vasculature, effect of indomethacin in utero, guinea pig. 693

sepsis and meningitis, immunoglobulin effect, rat, 455

serum melatonin manipulation. calcemic responses, rat, 414

short apneas, types and frequency, developmental study, 344

short chain fatty acid profile, fecal water analysis, colon, pig. 720

tachypnea of unknown cause, lung function in, 581

upper intestinal mucosa, milk feed composition and, guinca pig, 675

ventilation pressure, effect on cortical subarachnoid cerebrospinal prostanoids. piglet, 647

head position alteration, brain oxidative phosphorylation after, 302

Nephrotic syndrome, steroid responsive, inhibition of lipoprotein lipase, 197

Neutrophil

Chediak-Higashi syndrome, normal-sized lysosomes in, 208

chemotaxis, betamethasone effect, neonate, 150

Nutrition

deprivation, biochemical determinants of growth sparing during, neonate, 599

enhancement, biochemical determinants of growth sparing during, neonate, 599

intravenous supplementation, fetal, effect on growth retardation, sheep, 55

Obesity, erythrocyte fluidity, changes studied, 141

Ornithine, effect on creatine excretion, hyperornithinemia-hyperammonemiahomocitrullinuria syndrome, 364

Ornithine decarboxylase, biochemical determinants of growth sparing and, during nutritional deprivation or enhancement, neonatal brain and heart, 599
Oxygen

delivery, cyanotic heart disease, neonatal lamb, 389

delivery and consumption, during streptococcal group B sepsis, piglet. 509

metabolism, hypoxemia effects, lamb, 177

retinopathy induced by, effects of variable oxygenation during recovery phase, kitten, 297

transport, hypoxemia effects, lamb, 177

Oxygen free radical, asphyxia treatment, cerebral survival after, newborn sheep, 62

Pancreas

DNA and protein synthesis in, rats, 34

insufficiency, detected by cholesteryl octanoate breath test, rat, 257

Patent ductus arteriosus, additional volume loads and, premature lamb, 53

Peptide

trial natriuretic, action in immature ovine kidney, 11

cereal prolamin, role of mannan in prevention of intestinal damage induced by, rat fetus, 703

Pertussis toxin, immunization, anaphylactic reaction, mouse, 262

Phagocytosis, nonopsonic, susceptibility of Pseudomonas aeruginosa, cystic fibrosis, 429

Phosphatidylcholine, saturated, glycogen and, fetal lung type II cells, rat, 79

Placenta, glucose utilization, effect of insulin glucose, and ketone bodies, rat, 483

Plasma, $\alpha 1$-protease inhibitors, fetal-specific forms, mice,

Pneumococci, impaired pulmonary clearance, neonatal rat. 736

Polyamines, biochemical determinants of growth sparing and, during nutritional deprivation or enhancement, neonatal brain and heart, 599

Polypeptide, atrial natriuretic, ontogeny and characterization, fetal rat. 115

Polysaccharide, antigens, impaired natural antibody response to, cystic fibrois, 708

Pregnancy, late, uterine metabolism during, pig, 587

Prematurity

docasahexaenoic and eicosapentaenoic acid uptake, related to intake and mode of administration, 292

exogenous surfactant distribution, ventilation methods compared, lamb. 725

fat absorption, improved by taurine supplementation of premature formula 67

laryngeal muscle activity, control of, 87

patent ductus arteriosus, additional volume loads and, lamb, 531

pulmonary compliance, betamethasone effect, rabbit, 730

short apneas, types and frequency, 344

vancomycin pharmacology, seriously ill infants, 360

Pressure

arterial

response of fetal ventricles, lamb, 621 small pulmonary wedge

effect of alveolar hypoxia, awake lamb, 679

Prolidase, monoclonal and polyclonal antibodies in. immunochemical studies, 627

Propafenone hydrochloride, electrophysio- logic effects, neonatal heart, dog, 50

Prostanoids, ventilation pressure effect, cortical subarachnoid cerebrospinal fluid, newborn pig, 647

Protease inhibitor, $\alpha 1$-, fetal-specific forms, mouse plasma, 1

Protein

calcium-binding, variations in dietary calcium and, 518

enzyme, absence, in tyrosinemia, 394

neonatal deficiency, lung development impaired by, rat, 45

synthesis

DNA and, developing rat pancreas, 34

ontogenetic changes in rates of, fetus, 688

transport, during rotavirus diarrhea, mice, 72

Proteoglycan, cartilage, structure and abundance, early development of human fetus, 409

Pseudomonas aeritginosa

chronic pulmonary infection, mouse, 698

colonization, correlation of serum opsonic activity with, cystic fibrosis, 383

mucoid, phagocytosis resistance of, 429

pulmonary infection, immunohistological evaluation, cystic fibrosis, 743

Pseudomonas cepacia, chronic pulmonary infection, mouse, 698

Puberty, corticotropin-releasing factor, adrenocorticotropin, cortisol, and dehydroepiandrosterone response to, 41

Pyruvate dehydrogenase, first component, systemic deficiency, 312

Respiration

frequency and variability, sudden infant death syndrome victims, 158

pauses during, heart rate variability during, puppies and dogs, 306

Retinopathy, oxygen-induced, kitten, 297

Reye's syndrome, pathogenesis, mitochondrial toxicity of margosa oil, rat, 184

Rotavirus, diarrhea, protein transport during, mice, 72

Salbutamol, respiratory mechanics and, infants with bronchiolitis, 83

Saliva, local antibodies to $\alpha$-casein and $\beta$ lactoglobulin in, infants, 399

Sepsis

Escherichia coli, immunoglobulin effect, neonatal rat, 455

group B streptococcal

oxygen delivery, consumption, and metabolic acidosis during, piglet, 509

pulmonary function, effect of cyclooxygenase and lipoxygenase products, piglet, 478

Septicemia, meningococcal, C3 converting factor and, case report, 123

Serum, growth-promoting activity, normal and hypotrophic fetuses, 39

Skin, fibroblasts, catabolism of branchedchain L-amino acids in, 591

Sleep, arousal from, during rapidly developing hypoxemia, lamb, 471

Small intestine

alterations, after cholesterol deprivation, piglet, 330

cellular adaptation, after proximal enterectomy, rats, 29

fetal, in vitro damage prevented by mannan and other sugars, rat, 703

glycosylation, developmental changes, rat, 250 
Society for Pediatric Research, presidential address, 755

Somatomedin, immunocytochemistry, human fetus. 245

\section{Streptococcus}

group B

sepsis, oxygen delivery. consumption. and metabolic acidosis during. piglet. 509

sepsis, pulmonary function, piglet. 478

Sudden infant death syndrome, respiratory frequency and variability, in high risk victims, 158

Sulfisoxazole, effect on cerebral metabolism. newborn piglet. 1.35

Surfactant, exogenous, distribution. ventilation methods compared, premature lamb, 725

Sweat, hyposecretion. cystic fibrosis heterozygotes. 271
Tachypnea, unknown cause. lung function in. neonate. 581

Taurine

supplementation of premature formula, fat absorption improved by, preterm infants. 67

transport, maturational differences. rat kidney. 163

Transforming growth factor. possible role in ovine fetal development. 609

Tuberculin. transfer of immunity. mother to infant. 277

Tyrosinemia. type I. absence of enzyme protein in. 394

Utrasonography. transmission. bone status assessment. neonate. 541

Uterus. metabolism. late pregnancy. pig. 587

Vancomycin, pharmacology. seriously ill pre- mature infants, 360

Vasodilation. renal p-adrenoceptor-mediated. ontogens. endogenous catecholamines compared, sheep. 465

Ventilation

in utero. response of fetal ventricks, lamb. 621

prematurity. exogenous surfactant distribution. comparison of methods. lamb. 725

pressure, effect on cortical subarachnoid cerebrospina! prostanoids, newborn pigs. 647

Vitamin E. disposition in the eve, cats. 16 Vitamin $\mathrm{K}_{1}$

human milk content. stage of lactation and. 513

sister chromatid exchange indued by, human leukocytes and fetal siker cells. 405 